\title{
Applications of Hankel and Regular Matrices in Fourier Series
}

\author{
Abdullah Alotaibi ${ }^{1}$ and M. Mursaleen ${ }^{2}$ \\ ${ }^{1}$ Department of Mathematics, King Abdul Aziz University, P.O. Box 80203, Jeddah 21589, Saudi Arabia \\ ${ }^{2}$ Department of Mathematics, Aligarh Muslim University, Aligarh 202002, India
}

Correspondence should be addressed to M. Mursaleen; mursaleenm@gmail.com

Received 19 September 2013; Accepted 18 November 2013

Academic Editor: Adem Kılıçman

Copyright (C) 2013 A. Alotaibi and M. Mursaleen. This is an open access article distributed under the Creative Commons Attribution License, which permits unrestricted use, distribution, and reproduction in any medium, provided the original work is properly cited.

Recently, Alghamdi and Mursaleen (2013) used the Hankel matrix to determine the necessary and suffcient condition to find the sum of the Walsh-Fourier series. In this paper, we propose to use the Hankel matrix as well as any general nonnegative regular matrix to obtain the necessary and sufficient conditions to sum the derived Fourier series and conjugate Fourier series.

\section{Introduction and Preliminaries}

Let $X$ and $Y$ be two sequence spaces and let $A=\left(a_{n k}\right)_{n ; k=1}^{\infty}$ be an infinite matrix of real or complex numbers. We write $A x=\left(A_{n}(x)\right)$ provided that $A_{n}(x)=\sum_{k} a_{n k} x_{k}$ converges for each $n$. A sequence $x=\left(x_{k}\right)$ is said to be $A$-summable to $L$ if $\lim _{n} A_{n}(x)=L$. If $x=\left(x_{k}\right) \in X$ implies that $A x \in Y$, then we say that $A$ defines a matrix transformation from $X$ into $Y$ and by $(X, Y)$ we denote the class of such matrices. If $X$ and $Y$ are equipped with the limits $X$-lim and $Y$-lim, respectively, $A \in(X, Y)$ and $Y-\lim _{n} A_{n}(x)=X-\lim _{k} x_{k}$ for all $x \in X$, then we say that $A$ is a regular map from $X$ into $Y$ and in this case

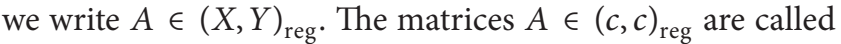
regular, where $c$ denotes the space of all convergent sequences.

The following are well-known Silverman-Töeplitz conditions for regularity of $A$.

Lemma 1. $A=\left(a_{n k}\right)_{n, k=1}^{\infty}$ is regular if and only if

(i) $\|A\|=\sup _{n} \sum_{k}\left|a_{n k}\right|<\infty$,

(ii) $\lim _{n} a_{n k}=0$ for each $k$,

(iii) $\lim _{n} \sum_{k} b_{n k}=1$.

A Hankel matrix is a special case of the regular matrix; that is, if $a_{n k}=h_{n+k}$ then the matrix is known as the Hankel matrix. That is, a Hankel matrix is a square matrix (finite or infinite), constant on each diagonal orthogonal to the main diagonal. Its $(n, k)$ th entry is a function of $n+k$. The Hankel transform of the sequence $x=\left(x_{k}\right)$ is defined as the sequence $y=\left(y_{n}\right)$, where $y_{n}=\sum_{k=0}^{\infty} h_{n+k} x_{k}$ provided that the series converges for each $n=0,1,2, \ldots$. An operator $T$ which transforms $x$ into $y$ as described is called the operator induced by the Hankel matrix $H$. In [1] we can find the applications of Hankel operators to approximation theory, prediction theory, and linear system theory. Hankel matrices have a number of applications in various fields.

Recently, Al-Homidan [2] proved that Hankel matrices are regular and obtained the sum of the conjugate Fourier series under certain conditions on the entries of Hankel matrix. Most recently, Alghamdi and Mursaleen [3] proved that Hankel matrices are strongly regular. Strongly regular matrices are those matrices which transform almost convergent sequences into convergent sequences leaving the limit invariant [4].

Our aim here is to find necessary and sufficient conditions for Hankel matrix as well as any arbitrary nonnegative regular matrix to sum the derived Fourier series and conjugate Fourier series.

\section{Main Results}

Let $f$ be $L$-integrable and periodic with period $2 \pi$, and let the Fourier series of $f$ be

$$
\frac{1}{a_{0}}+\sum_{k=1}^{\infty}\left(a_{k} \cos k x+b_{k} \sin k x\right) .
$$


Then the series conjugated to it is

$$
\sum_{k=1}^{\infty}\left(b_{k} \cos k x-a_{k} \sin k x\right)
$$

and the derived series is

$$
\sum_{k=1}^{\infty} k\left(b_{k} \cos k x-a_{k} \sin k x\right) .
$$

Let $S_{n}(x), \widetilde{S}_{n}(x)$, and $S_{n}^{\prime}(x)$ denote the partial sums of series (1), (2), and (3) respectively. We write

$$
\begin{gathered}
\psi_{x}(t)=\psi(f, t)= \begin{cases}f(x+t)-f(x-t), & \text { for } 0<t \leq \pi ; \\
g(x), & \text { for } t=0,\end{cases} \\
\beta_{x}(t)=\frac{\psi_{x}(t)}{4 \sin (1 / 2) t},
\end{gathered}
$$

where $g(x)=f(x+0)-f(x-0)$.

We propose to prove the following results.

Theorem 2. Let $f(x)$ be a function integrable in the sense of Lebesgue in $[0,2 \pi]$ and periodic with period $2 \pi$. Let $H=\left(h_{n+k}\right)$ be a Hankel matrix. Then for each $\beta_{x}(t) \in B V[0,2 \pi]$, the Hankel matrix transform of the sequence $\left(S_{k}^{\prime}(x)\right)$ is $\beta_{x}\left(0_{+}\right)$; that is,

$$
\lim _{n} \sum_{k=1}^{\infty} h_{n+k} S_{k}^{\prime}(x)=\beta_{x}(0+)
$$

if and only if

$$
\lim _{n \rightarrow \infty} \sum_{k=0}^{\infty} h_{n+k} \sin \left(k+\frac{1}{2}\right) t=0
$$

for every $t \in(0, \pi]$, where $B V[0,2 \pi]$ denotes the set of all functions of bounded variations on $[0,2 \pi]$.

In the next result, we replace the Hankel matrix by an arbitrary nonnegative regular matrix in the result of $\mathrm{Al}$ Homidan [2].

Theorem 3. Let $f(x)$ be a function integrable in the sense of Lebesgue in $[0,2 \pi]$ and periodic with period $2 \pi$. Let $A=$ $\left(a_{n k}\right)$ be a nonnegative regular matrix. Then A-transform of the sequence $\left(k \widetilde{S}_{k}(x)\right)$ converges to $g(x) / \pi$; that is

$$
\lim _{n} \sum_{k=1}^{\infty} k a_{n k} \widetilde{S}_{k}(x)=\frac{1}{\pi} g(x),
$$

if and only if

$$
\lim _{n \rightarrow \infty} \sum_{k=0}^{\infty} a_{n k} \cos k t=0
$$

for every $t \in(0, \pi]$, where each $a_{k}, b_{k} \in B V[0,2 \pi]$.

\section{Proofs}

We will need the following lemma which is known as the Banach Weak Convergence Theorem [5].

Lemma 4. $\lim _{n \rightarrow \infty} \int_{0}^{\pi} g_{n} d h_{x}=0$ for all $h_{x} \in B V[0, \pi]$ if and only if $\left\|g_{n}\right\|<\infty$ for all $n$ and $\lim _{n \rightarrow \infty} g_{n}=0$.

Proof of Theorem 2. We have

$$
\begin{aligned}
S_{k}^{\prime}(x) & =\frac{1}{\pi} \int_{0}^{\pi} \psi_{x}(t)\left(\sum_{m=1}^{k} m \sin m t\right) d t \\
& =-\frac{1}{\pi} \int_{0}^{\pi} \psi_{x}(t) \frac{d}{d t}\left(\frac{\sin (k+1 / 2) t}{2 \sin (t / 2)}\right) d t, \\
& =I_{k}+\frac{2}{\pi} \int_{0}^{\pi} \sin \left(k+\frac{1}{2}\right) t d \beta_{x}(t),
\end{aligned}
$$

where

$$
I_{k}=\frac{1}{\pi} \int_{0}^{\pi} \beta_{x}(t) \cos \frac{t}{2}\left(\frac{\sin (k+1 / 2) t}{\sin (t / 2)}\right) d t .
$$

Then

$$
\sum_{k=1}^{\infty} h_{n+k} S_{k}^{\prime}(x)=\sum_{k=1}^{\infty} h_{n+k} I_{k}+\frac{2}{\pi} \int_{0}^{\pi} L_{n}(t) d \beta_{x}(t),
$$

where

$$
L_{n}(t)=\sum_{k=1}^{\infty} h_{n+k} \sin \left(k+\frac{1}{2}\right) t .
$$

Since $\beta_{x}(t)$ is of bounded variation on $[0, \pi]$ and $\beta_{x}(t) \rightarrow$ $\beta_{x}(0+)$ as $t \rightarrow 0, \beta_{x}(t) \cos (t / 2)$ has also the same property. Hence by Jordan's convergence criterion for Fourier series $I_{k} \rightarrow \beta_{x}(0+)$ as $k \rightarrow \infty$.

Since the Hankel matrix $H=\left(h_{n+k}\right)$ is regular, we have

$$
\lim _{n} \sum_{k=1}^{\infty} h_{n+k} I_{k}=\beta_{x}(0+) .
$$

Now, it is enough to show that (6) holds if and only if

$$
\lim _{n} \int_{0}^{\pi} L_{n}(t) d \beta_{x}(t)=0
$$

Hence, by Lemma 4, it follows that (14) holds if and only if

$$
\left\|L_{n}(t)\right\| \leq M \quad \forall n, \forall t \in[0, \pi],
$$

and (6) holds. Since (15) is satisfied by Lemma 1(i), it follows that (14) holds if and only if (6) holds. Hence the result follows immediately.

Proof of Theorem 3. We have

$$
\begin{aligned}
\widetilde{S}_{n}(x) & =\frac{1}{\pi} \int_{0}^{\pi} \psi_{x}(t) \sin n t d t \\
& =\frac{g(x)}{n \pi}+\frac{1}{n \pi} \int_{0}^{\pi} \cos n t d \psi_{x}(t) .
\end{aligned}
$$


Therefore

$$
\sum_{k=1}^{\infty} k a_{n k} \widetilde{S}_{k}(x)=\frac{g(x)}{\pi} \sum_{k=1}^{\infty} a_{n k}+\frac{1}{\pi} \int_{0}^{\pi} K_{n}(t) d \psi_{x}(t),
$$

where

$$
K_{n}(t)=\sum_{k=1}^{\infty} a_{n k} \cos k t
$$

Now, taking limit as $n \rightarrow \infty$ on both sides of (17) and using Lemmas 1 and 4 as in the proof of Theorem 2, we get the required result.

Remark 5. If we take $a_{n k}=h_{n+k}$, then Theorem 2 is reduced to Theorem 4.1 of [2].

Remark 6. If we replace the matrix $H$ by an arbitrary nonnegative regular matrix $A=\left(a_{n k}\right)$ in Theorem 2, we get Theorem 1 of Rao [6].

\section{Conflict of Interests}

The authors declare that there is no conflict of interests regarding the publication of this paper.

\section{Acknowledgments}

This work was funded by the Deanship of Scientific Research (DSR), King Abdulaziz University, Jeddah, under Grant no. 130-072-D1434. The authors, therefore, acknowledge with thanks DSR technical and financial support.

\section{References}

[1] V. V. Peller, Hankel Operators and their Applications, Springer Monographs in Mathematics, Springer, New York, NY, USA, 2003.

[2] S. Al-Homidan, "Hankel matrix transforms and operators," Journal of Inequalities and Applications, vol. 2012, article 92, 2012.

[3] M. A. Alghamdi and M. Mursaleen, "Hankel matrix transformation of the Walsh-Fourier series," Applied Mathematics and Computation, vol. 224, pp. 278-282, 2013.

[4] J. P. King, "Almost summable sequences," Proceedings of the American Mathematical Society, vol. 17, pp. 1219-1225, 1966.

[5] S. Banach, Théorie des Operations Lineaires, Hafner, Warszawa, Poland, 1932.

[6] A. S. Rao, "Matrix summability of a class of derived Fourier series," Pacific Journal of Mathematics, vol. 48, pp. 481-484, 1973. 


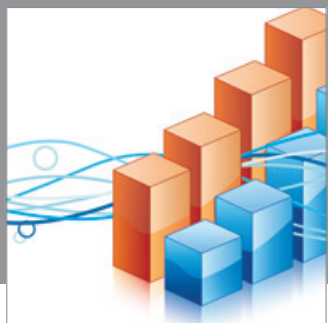

Advances in

Operations Research

mansans

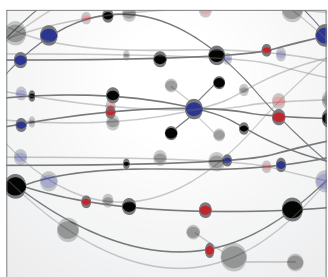

The Scientific World Journal
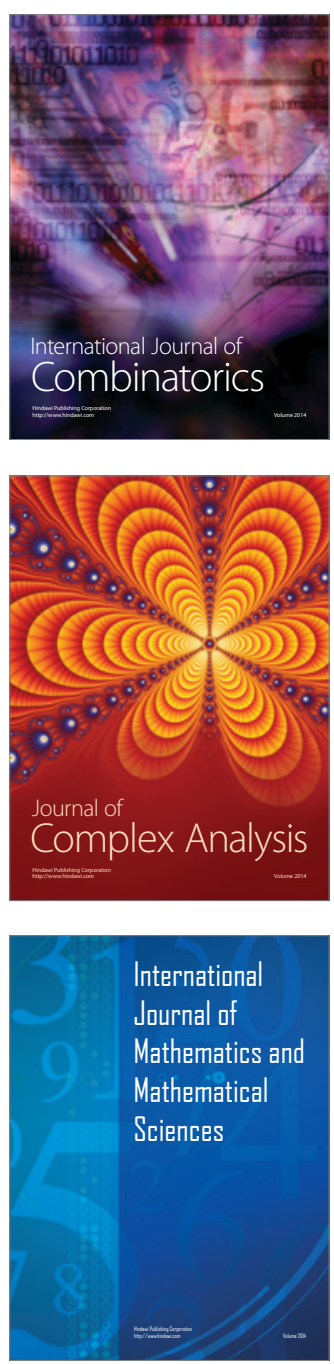
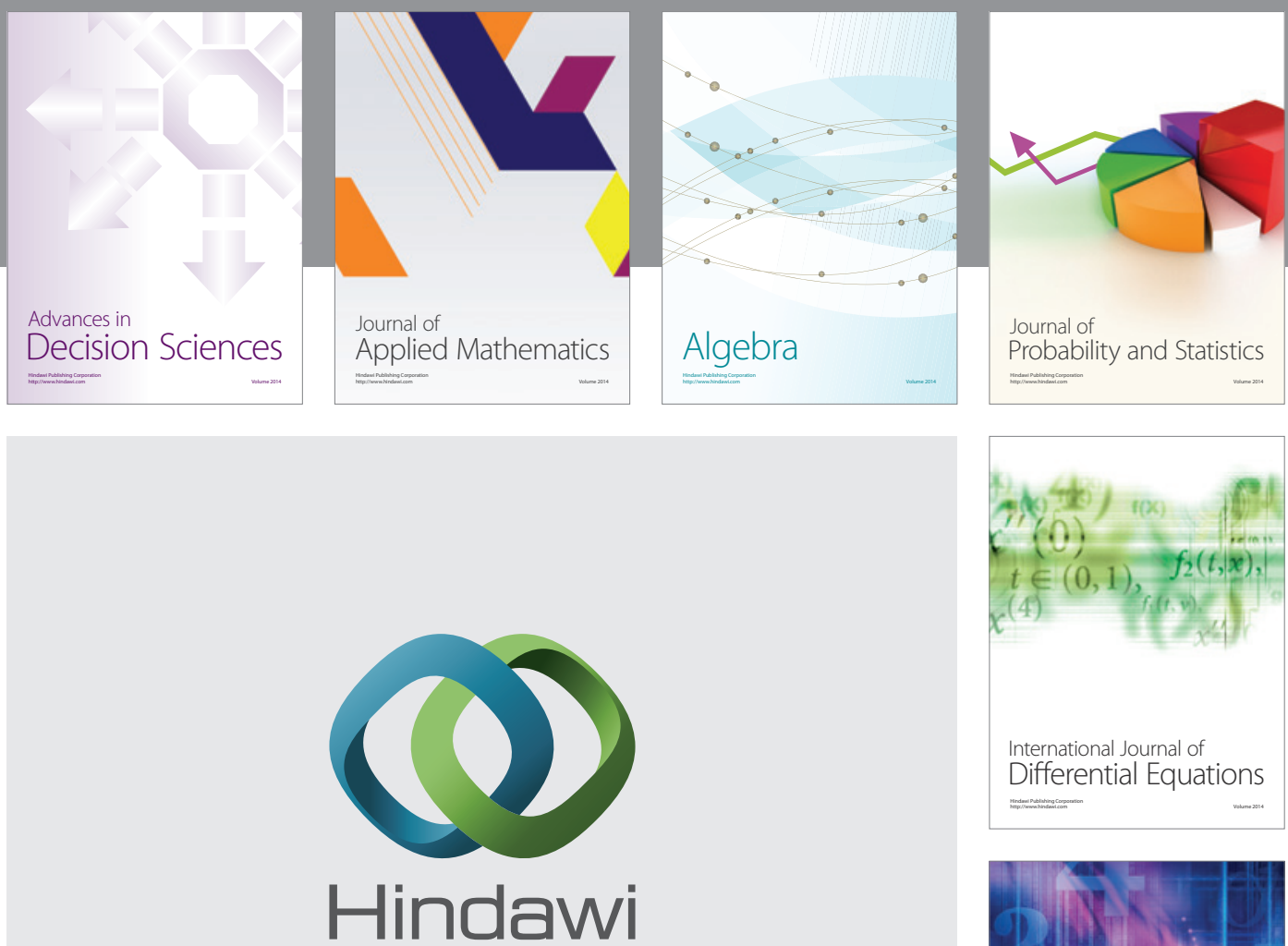

Submit your manuscripts at http://www.hindawi.com
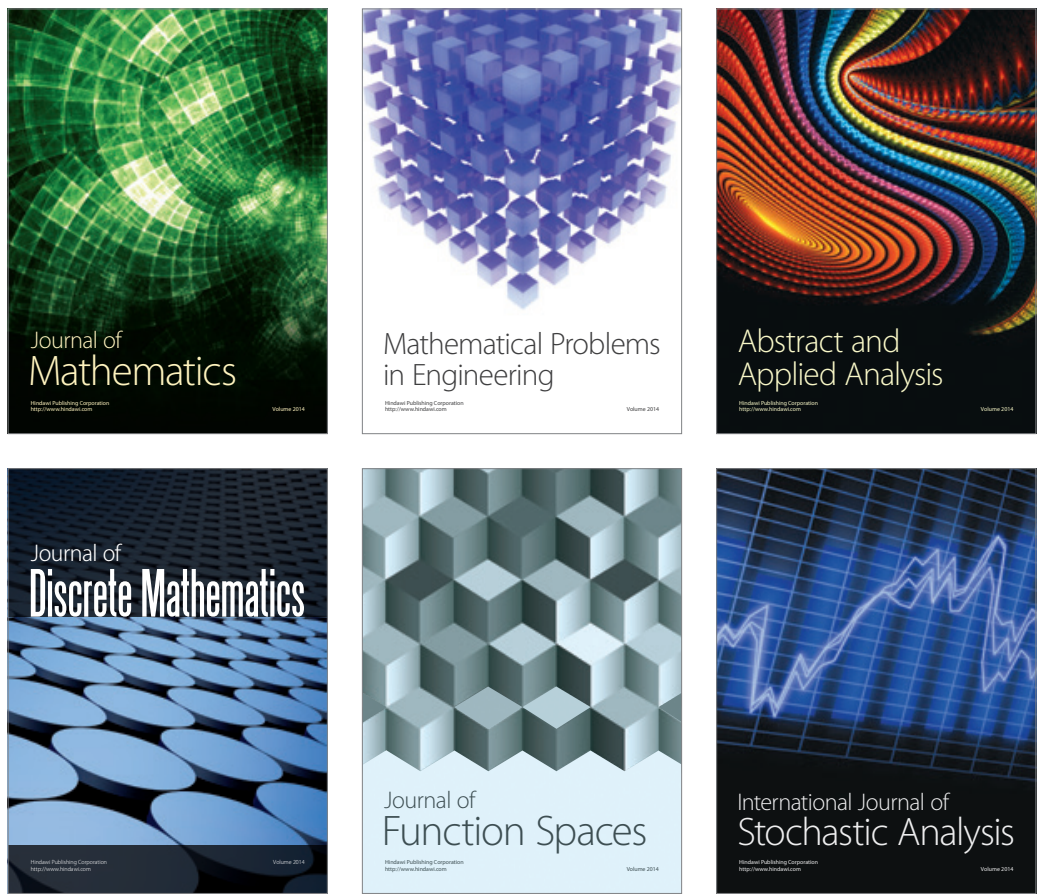

Journal of

Function Spaces

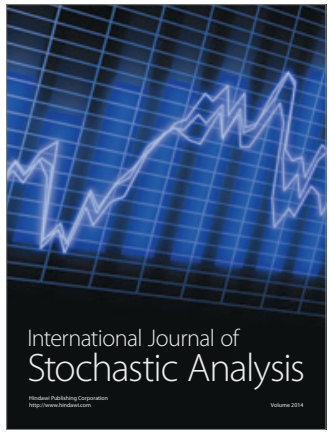

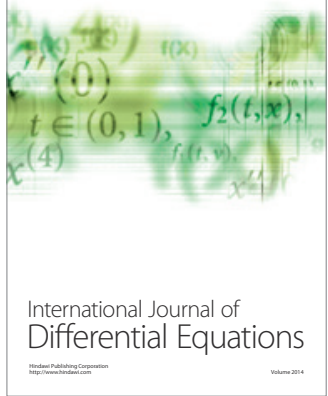
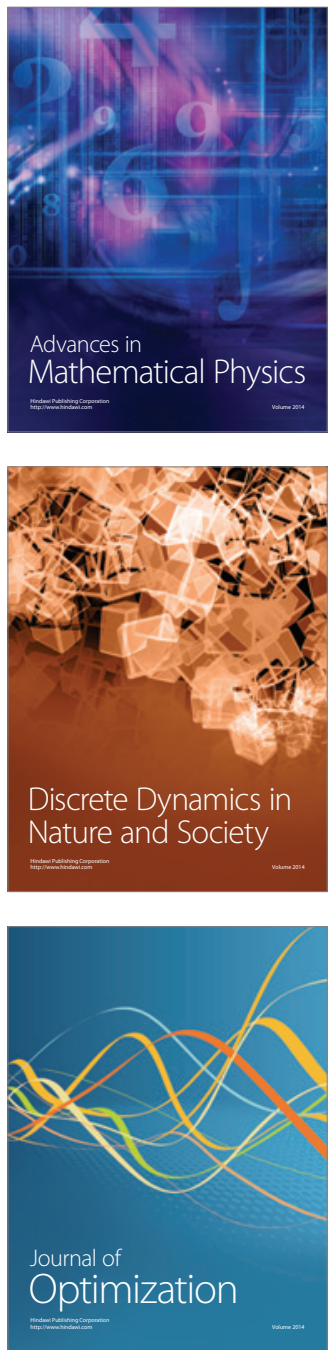